\title{
The Gaia astrometric survey for exoplanets in the solar neighborhood
}

\author{
Deborah Busonero ${ }^{1}$ \\ ${ }^{1}$ INAF - Osservatorio Astronomico di Torino \\ Via Osservatorio 20, 10025, Pino Torinese (TO), Italy \\ email: busonero@oato.inaf .it
}

\begin{abstract}
The Gaia astrometric mission holds the promise for crucial contributions to almost every subject of astrophysics and astronomy, including planetary systems astrophysics. We focus on the potential of the Gaia mission as perfect tool for a complete screening of nearby stars in search for exoplanets. We build our dissertation on the most recent results of the satellites astrometric payload performances and data reduction capabilities. We put the identified capabilities in context by illustrating the outstanding contribution to planetary sciences, in combination with nowadays and next decade exoplanets search programs, as a complement to other indirect and direct methods for the detection and characterization of planetary systems. We conclude by highlighting the crucial improvements in the optimization of the target lists of future dedicated observatory projects.
\end{abstract}

Keywords. astrometry, space vehicles: instruments, planetary systems

\section{Introduction}

The Gaia all-sky astrometric survey, due to launch in May 2013, will monitor, during its 5-yr nominal mission lifetime, all point sources (stars, asteroids, quasars, extragalactic supernovae, etc.) in the visual magnitude range $6-20 \mathrm{mag}$, for an amount of about $10^{9}$ objects. The final catalogue is foreseen for 2021.

Using the continuous scanning principle first adopted for Hipparcos, Gaia will determine the five basic astrometric parameters (two positional coordinates $\alpha$ and $\delta$, two proper motion components $\mu_{\alpha}$ and $\mu_{\delta}$, and the parallax $\varpi$ for all objects, with end-of-mission accuracy between $6 \mu$ as (at $\mathrm{V}=6 \mathrm{mag}$ ) and $200 \mu \mathrm{as}$ (at $\mathrm{V}=20 \mathrm{mag}$ ) for a G2V star.

Gaia astrometry, complemented by on-board spectrophotometry and (partial) radial velocity information (see de Bruijne et al. 2010), will have the precision necessary to quantify the early formation, and subsequent dynamical, chemical and star formation evolution of the Milky Way Galaxy. The broad range of crucial issues in astrophysics that can be addressed by the wealth of the Gaia data is summarized in Perryman (2005).

One of the relevant areas in which the Gaia observations will have great impact is the astrophysics of planetary systems, in particular when seen as a complement to other techniques for planet detection and characterization (e.g., Sozzetti 2009). This paper aims to be the first step towards a clarification about the Gaia astrometric performance and its contribution to the exoplanets research field, taking into account the most recent results on instrumental performances.

\section{The Gaia challenge}

The Gaia measurement concept requires observations in two lines of sight (LOS), separated by a large angle (named "Basic Angle" (BA) and set to 106.5), continuously 
scanning the sky along a great circle and completing a rotation in 6 hours, i.e., a scan rate of $60 \mathrm{arcsec} / \mathrm{s}$. The payload is composed of two identical telescopes with primary mirrors of $1.45 \times 0.5 \mathrm{~m}$ in size and $35 \mathrm{~m}$ nominal focal length, feeding a large common focal plane (FP): a CCD mosaic of about one hundred CCDs. The CCD array is divided in three region: the Sky Mapper-Astrometric Field (SM-AF), the Blue Photometer-Red Photometer (BP- RP), the Radial Velocity Spectrometer (RVS) devoted to astrometric, photometric, and spectroscopic measurements, respectively (Fig. 1 left panel). Each CCD operates in Time Delay Integration (TDI) mode matching the projected sidereal velocity (scanning velocity) with the CCD transfer velocity (the clock rate). The measurements repeatedly cover the whole sky, by the composition of rotation, precession, and orbital motion of the satellite. Gaia will collect 80 sky transits for each object on average in 5 years, with a dependency on where the satellite looks, ranging from few tenth to few hundred observations per object along the ecliptic.
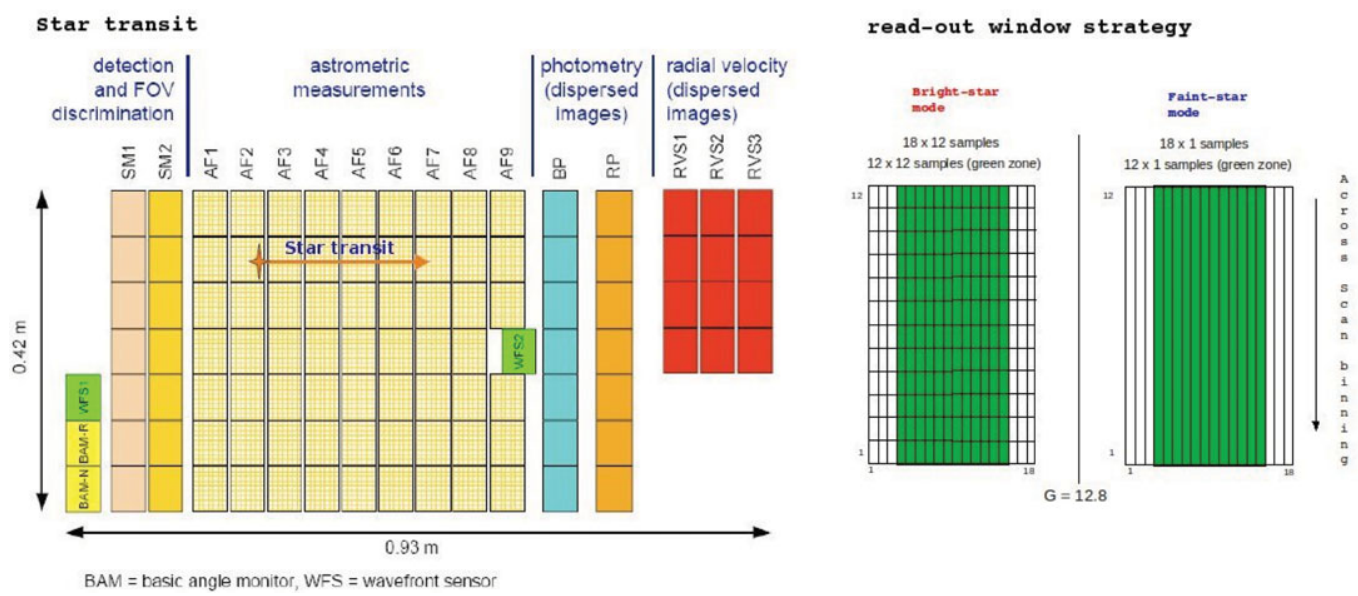

Figure 1. Gaia Focal Plane structure and read-out window strategy.

The instantaneous image is integrated throughout the whole transit over the CCD, as each logical pixel is generated at the leading edge and transferred in step with the corresponding point in object space up to the trailing edge of the device, where the readout process takes place. Readout on the Astrometric Field (AF) is restricted to the regions of interest identified by the SM in a way that for each star a window is selected for read-out according to the adopted windowing strategy (Fig. 1 right panel). The readout mode depends upon the target brightness, in particular using full-bidimensional image readout for stars brighter than $\mathrm{G}=12.8 \dagger$ and across scan binning (low resolution) to improve upon signal to noise ratio $(\mathrm{SNR})$ and reduce the data volume for stars fainter than $\mathrm{G}=12.8$, so that the output data is a one-dimensional signal. Time of observation and position on the sky are linked by the scan law plus the electro-optical response.

For each transit, a target is observed astrometrically in equal conditions on nine consecutive elementary exposures (the 9 AF CCDs). The independent composition of the elementary exposures provides the transit-level accuracy. In order to meet the end-ofmission accuracy goal, the location error at the level of the elementary exposure has to range from some tenth of as for the brightest stars $(G=6-10)$ to a few mas for $G=21$.

$\dagger$ Conversion rules from Johnson V to Gaia Astro G magnitude scale depends on star spectral type and for $\mathrm{A} 0 \mathrm{~V}$ is $\mathrm{G}=\mathrm{V}, \mathrm{B} 1 \mathrm{~V}$ is $\mathrm{G}=\mathrm{V}-0.02$, for $\mathrm{G} 2 \mathrm{~V}$ is $\mathrm{G}=\mathrm{V} 0.19$ and for $\mathrm{M} 6 \mathrm{~V}$ is $\mathrm{G}=\mathrm{V}$ 2.27 
This means that we need to maintain an elementary exposure accuracy on the location ranging from $10^{-4}$ to $10^{-2}$ of the along scan pixel size $(10 \mu \mathrm{m})$.

Gaia, like Hipparcos, is designed to be a self-calibrated instrument (Lindegren 2005), but PSF/LSF calibration, CCD calibration, radiation damage treatment, and transitlevel attitude diagnostics indipendent procedures are needed in the data reduction chain (Busonero et al. 2010) before starting the astrometric iterative solution (Hobbs 2008).

We don't enter here in the detail of the instrument calibration procedures. We narrow our dissertation down to instrument calibration accuracy and bright objects.

\section{Instrumental noise and bright stars}

The variation of the instrument response is quite unavoidable so its modeling is crucial to manage the effects that can affect the final micro-arcsecond level astrometric accuracy. Instrumental noise sources are divided in two different classes: random, i.e., photometric errors, and systematic errors. Systematic limitations are imposed by the CCD detectors, e.g., radiation damage (Short et al. 2010), gates, deviations from uniformity or from linear pixel response, and by optics imperfections, e.g., optical aberrations and distortions (Busonero et al. 2006), pixelization.

A critical item is the treatment of bright objects, which will constitute the bulk of the well-behaved celestial reference points utilized in the core processing and in the sphere recostruction. Operationally the term bright refers to objects of magnitude $\mathrm{G}<15.8$. The search for the best possible centroiding performance is of course critical for such stars. On the other hand, saturation starts at $\mathrm{G}=12.8$ and will become severe for those objects in the brightest magnitude bin of interest to Gaia (6-10).
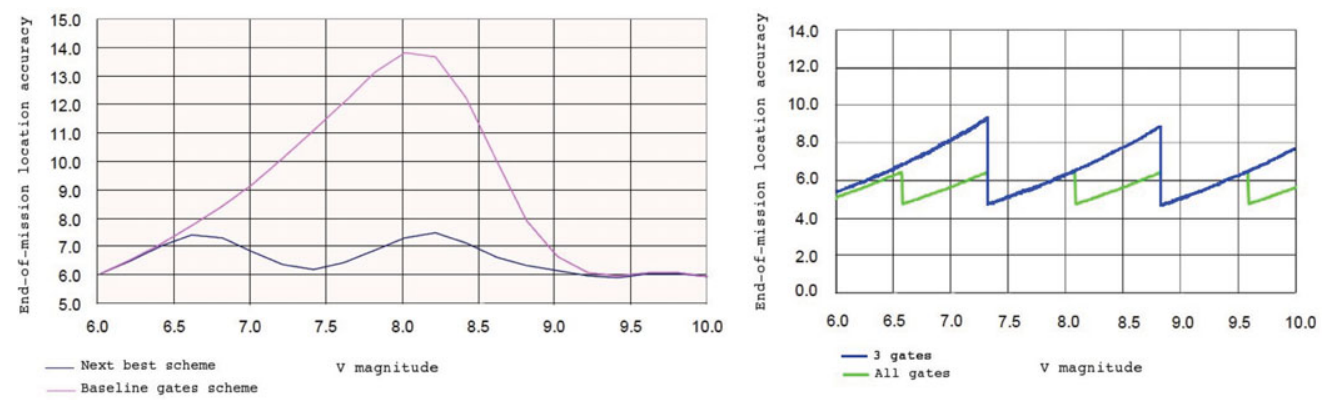

Figure 2. Gaia end-of-mission location accuracy in $\mu$ as depending on gates activation.(data derived from the "Science performance budget report", GAIA.ASF.RP.SAT.00005)

TDI gate activation is foreseen on board to prevent pixel saturation, i.e., allow the loss of photons to make the brighter stars behave like the fainter ones, thus maintaining similar centroiding performances over the whole bright magnitudes interval. But gates are also sources of instrumental noise since the CCDs have to be calibrated for each activated gate. It means that we need to find a reasonable trade-off among the possibility to avoid the saturation for all the transit objects, the possibility to perform the calibration of the gate catalog within a realistic calibration-block duration (within few weeks), and the limitations imposed by the VPU software which implements for one CCD row one TDI gates table for all CCDs strip and selects the TDI gate activation based on G magnitude, not on AC coordinate. It is a futher possible error source since the saturation level is not uniform over the CCD but has a significant (about 40\%) AC variation.

The Industry and the Gaia Scientific Consortium have studied several gates schemes. 
We show in Fig. 2 the comparison beetween four different gate schemes and the related performances. From the perspectives of the signal-to-noise of the CCD sample data of bright stars and centroiding precision the optimum solution would clearly be to use all 12 TDI gates available but the calibration needs impose a choice. Required gates calibration time becomes forbidding for a gates activation with more than seven gates as shown in Table 1 .

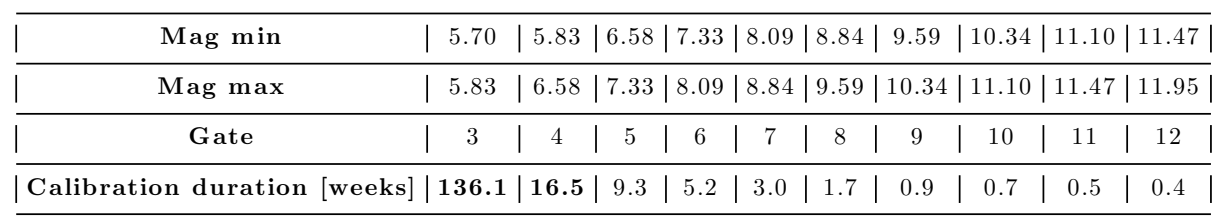

Table 1. Required gates calibration time in weeks for all gates activation.

The actual gates scheme counts six gates (Fig. 2 violet line) and provides an end-ofmission location accuracy for $7 \leqslant V \leqslant 9$ ranging from $7 \mu$ as to $14 \mu a s$. A new gates scheme with seven gates is under study (Fig. 2 blue line). It will provide an astrometric accuracy ranging between 6 and 7 mas. In Fig. 3 we show clearly how that degradation of the performances can significantly change the Gaia contribution to the exoplanets survey.

We have to keep in mind also that the radiation damage can introduce a similar degradation if not adeguately calibrated.

On the other hand, the saturation of the image core theoretically improves the centroiding performance through improved S/N on the slopes. Significantly better centroid errors can be achieved by dealing directly with saturated images, i.e., without (or very limited) actuation of the gates, but when an accurate calibration of the actual in-flight $\mathrm{PSF} / \mathrm{LSF}$ is made available. In this case the dealing with the radiation damage in presence of saturated samples could introduce an other source of errors.

\begin{tabular}{|c|c|c|c|c|c|c|}
\hline$\sigma_{\psi}^{a}(\mu \mathrm{as})$ & $N_{\star}{ }^{b}$ & $N_{\mathrm{d}}{ }^{c}$ & $N_{\mathrm{m}}^{d}$ & $N_{\mathrm{d}, \text { mult }}{ }^{\mathrm{e}}$ & $N_{\mathrm{m} \text {,mult }} f$ & $N_{\text {copl }^{g}}$ \\
\hline 11 & 500000 & 8000 & 4000 & 1000 & 500 & 159 \\
\hline 16 & 148148 & 2370 & 1185 & 296 & 148 & 47 \\
\hline 22 & 62500 & 1000 & 500 & 125 & 62 & 19 \\
\hline 27 & 18519 & 296 & 148 & 37 & 18 & 5 \\
\hline 60 & 4000 & 64 & 32 & 8 & 4 & 1 \\
\hline 100 & 500 & 8 & 4 & 1 & 0 & 0 \\
\hline
\end{tabular}

Figure 3. Gaia worse and better capability for $\mathrm{V}<13$ and $\mathrm{d}<200 p c$ depending on instrument calibration accuracy. (courtesy of Casertano et al. (2008))

\subsection{Transit-measurement accuracy}

We pointed out until now how the instrument calibration performances drive the Gaia science case, bringing specific research field like the exoplanets to disappear from the Gaia science case, if the transit-measurement precision degrades significantly,

In Table 2 we list the AF AL scan transit-level location estimation errors in units of ras as function of $\mathrm{V}$ and spectra types, taking into account the blue line gates scheme and the possibility of calibrating the image distortion due to the radiation damage so that cleaning the Gaia measurement. 


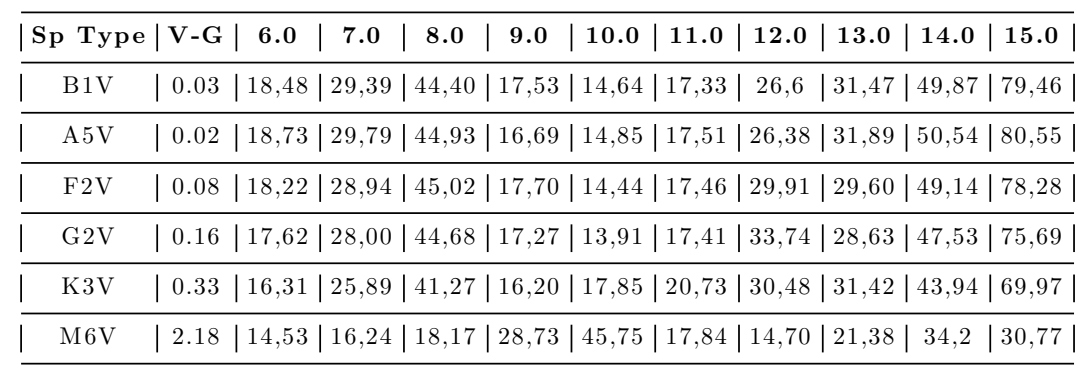

Table 2. AF AL scan transit-level location estimation errors in units of $\mu a s$ as function of $\mathrm{V}$ and spectral types, for unreddening stars.

\section{Contribution to exoplanets discoveries: Conclusion}

Gaia will be a great tool for a complete screening of nearby stars in search for exoplanets. Indeed for a position accuracy ranging from 10 to $15 \mu$ as it promises the monitoring of hundreds of thousands of FGK stars to $\sim 200 \mathrm{pc}$, performing a complete census of all stellar types with a dection limit set to $\sim 1 \mathrm{M}_{J}$ planets and $P<10$ years. About one thousand multiple systems will be measurable, giving relative inclinations and orbits for possibly several hundred systems. The newly discovered single-planets systems will amount to several thousands (Fig. 3).

Such an unbiased, magnitude-limited planet census of hundreds of thousands stars will be essential to study the statistics of planetary systems as a function of stellar properties (the statistics of exoplanets discoveries by astrometry to-date equals zero).

The Gaia exoplanets catalogue strenght lies also in the complementarity with respect to other indirect and direct methods for the detection and characterization of planetary systems, and in the crucial improvements in the optimization of the target lists of future dedicated observatory projects.

\section{Acknowledgement}

The Italian participation in the Gaia mission is supported by the Italian Space Agency, under contract ASI I/058/10/0. I thank Alessandro Sozzetti for useful discussions and careful proofreading of this manuscript.

\section{References}

Busonero, D., Gai, M., \& Lattanzi, M. G. 2010, in: Oschmann, J. M., Jr., Clampin, M. C., MacEwen, H. A. (eds.), Proc. SPIE, 7731, 33

Busonero, D., Gai, M., Gardiol, D., Lattanzi, M. G., \& Loreggia, D. 2006, Aछ A, 449, 827

Casertano, S., Lattanzi, M. G., Sozzetti, A., Spagna, A., Jancart, S., Morbidelli, R., Pannunzio, R., Pourbaix, D., \& Queloz, D. 2008, A\&A, 482, 699

de Bruijne, J., Kohley, R., \& Prusti, T. 2010, in: Oschmann, J. M., Jr., Clampin, M. C., MacEwen, H. A. (eds.), Proc. SPIE, 7731, 1

Hobbs, D., Lindegren, L., Holl, B., Lammers, U., \& O'Mullane, W. 2008, Proc. IAU Symp., 248, 268

Lindegren, L. \& de Bruijne, J. H. J. 2005, P. K. Seidelmann, A. K. B. Monet (eds.), ASP Conf. Ser., 338,25

Perryman, M. A. C.. 2005, in P. Kenneth Seidelmann and Alice K. B. Monet (eds.), 一textitASP Conf. Ser., 338, 3

Short, A., Prod'homme, T., Weiler, M., Brown, S. W., \& Brown, A. G. A.. 2010, in: Holland, A. D., Dorn, D, A. (eds.), Proc. SPIE, 7742, 12

Sozzetti, A. 2010, in I. F. Corbett, ed., Highlights of Astronomy, Proc. XXVIIth IAU General Assembly, 15, 716 